\title{
CTGF enhances resistance to 5-FU-mediating cell apoptosis through FAK/MEK/ERK signal pathway in colorectal cancer
}

This article was published in the following Dove Press journal:

OncoTargets and Therapy

30 November 2016

Number of times this article has been viewed

\section{Kai Yang \\ Kai Gao \\ Gui Hu \\ Yanguang Wen \\ Changwei Lin \\ Xiaorong $\mathrm{Li}$}

Department of General Surgery, The Third Affiliated Hospital of Central South University, Central South University, Changsha, Hunan, People's Republic of China
Correspondence: Xiaorong Li

Department of General Surgery,

The Third Affiliated Hospital of

Central South University, Central South

University, I 38 Tongzhipo Road, Hunan

Province, Changsha 4I00I3, People's

Republic of China

Tel +86 73I 886I 8232

Email lixiaorong@medmail.com.cn
Abstract: Colorectal cancer (CRC) is one of the most commonly diagnosed cancers among both males and females; the chemotherapy drug 5-fluorouracil (5-FU) is one of a doctors' first lines of defense against CRC. However, therapeutic failures are common because of the emergence of drug resistance. Connective tissue growth factor (CTGF) is a secreted protein that binds to integrins, and regulates the invasiveness and metastasis of certain carcinoma cells. Here, we found that CTGF was upregulated in drug-resistant phenotype of human CRC cells. Overexpression of CTGF enhanced the resistance to 5-FU-induced cell apoptosis. Moreover, downregulating the expression of CTGF promoted the curative effect of chemotherapy and blocked the cell cycle in the G1 phase. We also found that CTGF facilitated resistance to 5-FUinduced apoptosis by increasing the expression of B-cell lymphoma-extra large (Bcl-xL) and survivin. Then we pharmacologically blocked MEK/ERK signal pathway and assessed 5-FU response by MTT assays. Our current results indicate that the expression of phosphorylated forms of MEK/ERK increased in high CTGF expression cells and MEK inhibited increases in 5-FU-mediated apoptosis of resistant CRC cells. Therefore, our data suggest that MEK/ERK signaling contributes to 5-FU resistance through upstream of CTGF, and supports CRC cell growth. Comprehending the molecular mechanism underlying 5-FU resistance may ultimately aid the fight against CRC.

Keywords: connective tissue growth factor, 5-fluorouracil, mitogen-activated protein kinase/ extracellular regulated protein kinases, phosphatidyl inositol 3-kinase/serine/threonine kinase Akt, colorectal cancer

\section{Introduction}

Colorectal cancer (CRC) is one of the most common cancers in the People's Republic of China in terms of both morbidity and mortality. Although surgical resection has been the primary treatment modality, chemotherapy is still the important treatment for CRC. ${ }^{1}$ The chemotherapeutant, 5-fluorouracil (5-FU), causes cytotoxic damage by way of targeting the metabolism of RNA bases. It is largely used in the medical care of various kinds of cancers, including colorectal, bladder, lung, and liver cancers. ${ }^{2}$ However, the clinical responses to 5-FU vary greatly, and chemoresistance is a major reason for CRC therapy failure. Therefore, it is very important to find molecular markers that modulate CRC chemoresistance. ${ }^{3}$

Connective tissue growth factor (CTGF [CCN2]) is an extracellular matrix (ECM)associated molecule belonging to the family of growth factors termed the $\mathrm{CCN}$, which contains cysteine-rich 61 (CCN1), CTGF/CCN2, nephroblastoma overexpressed (CCN3), Wnt-inducible secreted protein-1 (CCN4), rCop1 (CCN5), and Wisp-3 
(CCN6). The CNN gene family is characterized by very high levels of amino acid sequence identity ranging from $50 \%$ to $90 \%$. All members of the CCN gene family are secretory proteins. Each includes four obvious structural modules: insulin-like growth factor-binding protein, von Willebrand type $\mathrm{C}$, thrombospondin 1, and $\mathrm{COOH}$-terminal cysteine-rich domain (CT). Each module shows homology to conserved domains in several forms of ECM proteins. These modules participate in protein binding and contain conserved disulfide bridges. ${ }^{4}$ They have been known to influence several important cellular functions, such as cell cycle regulation, apoptosis, ECM production, angiogenesis, and migration.

Of late, there is accumulating evidence that CTGF expression is highly correlated with the development of cancers. ${ }^{5}$ Elevated CTGF expression has been observed in mammary carcinomas, ${ }^{6}$ spongioblastoma, ${ }^{3}$ thyroid cancers, ${ }^{7}$ and CRC. ${ }^{8}$ Taking into account that CTGF performs a significant role in tumor metastasis and that metastatic cancerous cells usually exhibit resistance to apoptosis, we postulated that CTGF expression would increase drug resistance in CRC cells. It is still unclear whether CTGF expression would deregulate apoptosis modulators or would confer drug tolerance in CRC cells. We herein studied the influence of CTGF on the survival and chemosensitivity of CRC cells and the signaling pathways involved.

\section{Materials and methods}

\section{Cell culture}

All the cell lines (NCM460, HT-29, SW620, HCT116, and LOVO) were purchased from IMCAS-BRC (Shanghai, People's Republic of China) and China Center of Industrial Culture Collection (Beijing, People's Republic of China). NCM460 and HT29 cells were maintained in Roswell Park Memorial Institute 1640 (Thermo Fisher Scientific, Waltham, MA, USA) with 10\% fetal bovine serum (FBS; Thermo Fisher Scientific); SW620 cells were maintained in Dulbecco's Modified Eagle's Medium (Thermo Fisher Scientific) with 20\% FBS; HCT116 and LOVO cells were maintained in DMEM with 10\% FBS. All cells were maintained in $1 \%$ penicillin/streptomycin and cultured in humidified incubators at $37^{\circ} \mathrm{C}$ with $5 \% \mathrm{CO}_{2}$.

To establish the drug-resistant cell subline HT-29/5-FU (HT-29-FU) and SW620/5-FU (SW620-FU), HT-29 and SW620 cells were exposed to stepwise increase of 5-FU (Sigma-Aldrich, St Louis, MO, USA) concentrations from 10 to $200 \mu \mathrm{M}$. When no significant cell deaths were noted after 5-FU treatment, the cells were checked by a cell survival assay (MTT) in the presence of 5-FU.

\section{Construction and transfection of recombinant expression plasmids}

The complete CTGF open reading frame was amplified by polymerase chain reaction (PCR). PCR amplification was performed in $0.2 \mathrm{mM}$ dNTPs, $1.5 \mathrm{mM} \mathrm{MgCl}$, $40 \mathrm{U} / \mathrm{mL}$ of Pfu DNA polymerase (Thermo Fisher Scientific), and 1 nmol of each PCR primer (sense: CCCAAGCT TATGACCGCCGCCAGTATGG, and antisense: CCGG AATTCTCATGCCATGTCTCCGTACATCTT), which were designed to amplify the full-length CTGF cDNA. PCR products were purified from agarose gels using the Viogene gel/PCR DNA Isolation System (Viogene, Sunnyvale, CA, USA). The complete CTGF was cloned into the topoisomerase-activated pcDNA3.1 vector (Invitrogen, Carlsbad, MA, USA).

Cells were plated in antibiotic-free media. The next day, the cells were transfected using Lipofectamine 2000 (Invitrogen) with pcDNA3.1, pcDNA3.1-CTGF, CTGFsiRNA Sc-39329; Santa Cruz Biotechnology, Dallas, TX, USA or control siRNA Sc-37007; Santa Cruz Biotechnology according to the manufacturer's protocol, respectively.

\section{MTT assay}

Cell viability was measured by MTT method. Cells were seeded at a concentration of 5,000 cells per well in 96-well plates. After treatment with 5-FU for 48 hours, the cultures were washed with phosphate-buffered saline three times. Subsequently, $0.5 \mathrm{mg} / \mathrm{mL}$ MTT solution was added to each well and incubated at $37^{\circ} \mathrm{C}$ for 30 minutes. In order to dissolve formazan crystals, the culture medium was replaced with equivoluminal dimethyl sulfoxide. While mixing and shaking the whole sample at $25^{\circ} \mathrm{C}$ for 15 minutes, absorbance of each well was detected with a microplate reader at $550 \mathrm{~nm}$.

\section{Western blotting analysis}

Cells were lysed in radioimmunoprecipitation assay buffer, which was supplemented with protease and phosphatase inhibitors. Protein extracts were resolved by sodium dodecyl sulfate polyacrylamide gel electrophoresis and transferred to nitrocellulose membranes. After blocking with $4 \%$ bovine serum albumin for 1 hour at room temperature, the blots were incubated with primary antibodies (focal adhesion kinase [FAK; Abcam, Cambridge, MA, USA, Immunoway, YM0260, 1:1,000], p-FAK [Immunoway, YP1025, 1:500], MEK [Santa Cruz Biotechnology, Sc-6250, 1:500], p-MEK [Santa, Sc-271914, 1:200], ERK1/2 [Immunoway, YT1626, 1:200], p-ERK1/2 [Immunoway, YP1197, 1:500], P13K 
[Abcam, Ab86714, 1:500], AKT [Assay, C10531, 1:200], p-AKT [Immunoway, YP0590, 1:200], FOXM1 [Abcam, Ab175798, 1:1,000], RhoA [Abcam, Ab68826, 1:1,000], B-cell lymphoma-extra large [Bcl-xL; Abcam, Ab32370, 1:200], Survivin [Abcam, Ab24479, 1:200], CTGF [Proteintech, Rosemont, IL, USA, 23903-1-AP, 1:100]) for overnight. $\beta$-actin monoclonal AC-15 (Sigma-Aldrich) at 1:10,000 was used as a loading control. Odyssey Imaging System and ImageQuant software (Beckman Coulter Inc., Brea, CA, USA) was used to visualize the blots and obtain the quantitative data.

\section{Quantitative real-time PCR}

Total RNA was extracted using the RNeasy purification kit (Qiagen, Valencia, CA, USA) and treated with DNase (Invitrogen). The reverse transcription reaction was performed using $2 \mu \mathrm{g}$ of total RNA that was reverse transcribed into cDNA using random primers. A total of $100 \mathrm{ng}$ cDNA was added per $25 \mu \mathrm{L}$ reaction, along with sequence-specific primers and Taq-Man probes. Sequences for target gene primers (sense: CTCCTGCAGGCTAGAGAAGC, and antisense: GATGCACTTTTTGCCCTTCTT) and probes were purchased commercially ( $\beta$-actin was used as the internal control; Applied Biosystems, Carlsbad, CA, USA). Quantitative PCR assays were carried out in triplicate using a StepOnePlus sequence detection system. ${ }^{7,8}$

\section{Cell cycle analysis and apoptosis detection}

Cells were harvested for cell cycle analysis. Using Dulbecco's phosphate-buffered saline (DPBS) $+10 \%$ FBS, cells were washed twice, fixed in ice-cold $80 \%$ ethanol, and stored at $-20^{\circ} \mathrm{C}$ for at least 24 hours. Fixed cells were incubated in $50 \mu \mathrm{L}$ of propidium iodide buffer $(20 \mu \mathrm{g} / \mathrm{mL}$ propidium iodide [Sigma-Aldrich], 0.1\% Triton-X 100, $200 \mu \mathrm{g} / \mathrm{mL}$ RNaseA [Promega Corporation, Fitchburg, WI, USA] in DPBS) for 30 minutes in the dark. The cells were then resuspended in $400 \mu \mathrm{L}$ of DPBS for flow analysis.

\section{Statistics}

All statistical analyses were performed using the SPSS (version 17.0; SPSS Inc., Chicago, IL, USA) and GraphPad software (GraphPad Software, Inc., La Jolla, CA, USA). The values given are mean \pm standard error of the mean (SEM). $P$-values were determined for experimental versus control treatments by two-tailed Student's $t$-test, ${ }^{*} P<0.05$, $* * P<0.01, * * * P<0.001$.

\section{Results \\ 5-FU increases CTGF expression in CRC cells}

CTGF has been shown to play an important role in CRC cancer metastasis and is also associated with increase in tumor size. Because highly metastatic cancer cells exhibit a drug-resistant phenotype, we speculated that CTGF expression may be involved in promoting drug resistance in CRC cells. To investigate the effect of CTGF expression and the chemotherapy response in CRC cells, we first established HT-29-FU (5-FU/HT-29) and SW620-FU (5-FU/SW620) cell lines from parental HT-29 and SW620 cells and checked them by MTT assay. The results showed the $50 \%$ inhibitive concentration $\left(\mathrm{IC}_{50}\right)$ values of HT-29 and HT-29-FU were 14.21 and $66.71 \mu \mathrm{M}$, respectively, and the resistance index was 4.69 (Figure 1A); the $\mathrm{IC}_{50}$ values of SW620 and SW620-FU were 17.46 and $67.86 \mu \mathrm{M}$, respectively, and the resistance index was 3.88 (Figure 1A). This indicated that HT-29-FU and SW620-FU cell lines were established successfully. Furthermore, we examined CTGF expression of CRC cell lines (HT-29 and SW620) and 5-FU-resistant CRC cell lines (HT-29-FU and SW620-FU) with Western blot and quantitative PCR. The protein and mRNA expression of CTGF were significantly increased in 5-FU-resistant CRC cell lines compared with their parent cells (Figure 1B and C). These findings suggested that CTGF is upregulated during chemotherapy in CRC cells.

\section{Overexpression of CTGF enhances resistance to 5-FU-promoting cell death}

To examine the potential role of CTGF on the regulation of chemoresistance to 5-FU, we first confirmed that the pCDNA3.1-CTGF vector and CTGF small interfering RNA (CTGF-siRNA) were overexpressing and interfering with the protein and mRNA of CTGF in HT-29 and SW620 cells detected by Western blot and quantitative PCR (Figure 2A-E). Previous studies have demonstrated that CTGF expression confers resistance to chemotherapeutants in glioblastoma ${ }^{9}$ and ovarian cancer. ${ }^{10}$ Then, in our study, we detected the cell viability of HT-29-FU and SW620-FU cells by MTT assay when HT-29-FU and SW620-FU cells were transfected with pCDNA3.1-CTGF and CTGF-siRNA vectors. The $\mathrm{IC}_{50}$ values of 5-FU were examined. Overexpression of CTGF significantly enhanced the $\mathrm{IC}_{50}$ values (HT-29-FU: 62.97-86.77 $\mu \mathrm{M}$; SW620-FU: 66.46-89.50 $\mu \mathrm{M}$ ), and silencing of CTGF dramatically reduced the $\mathrm{IC}_{50}$ values (HT-29-FU: 59.90-43.24 $\mu \mathrm{M}$; SW620-FU: 62.87-47.05 $\mu \mathrm{M}$ ). 
A

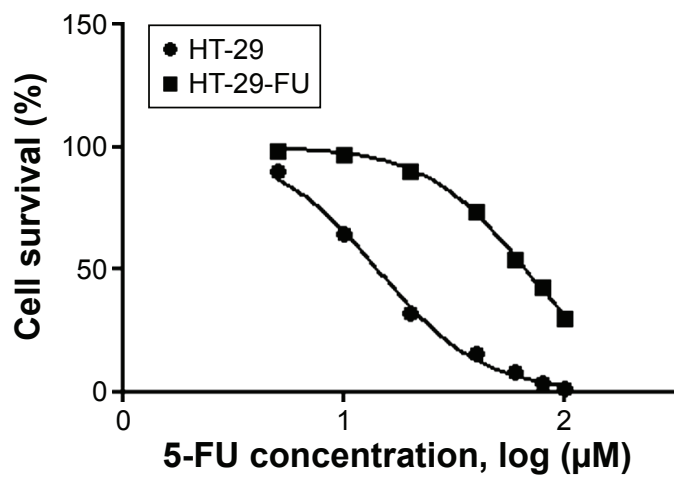

B
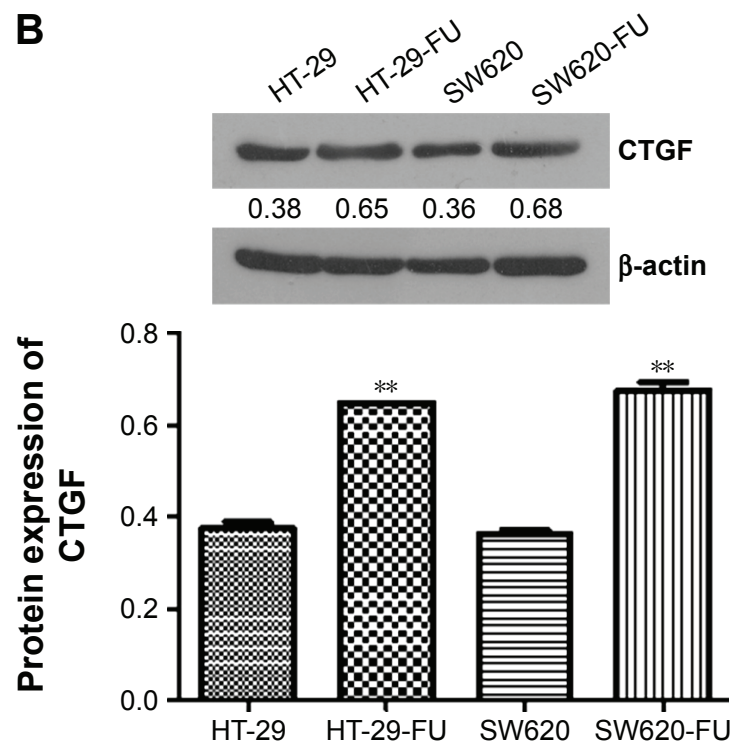

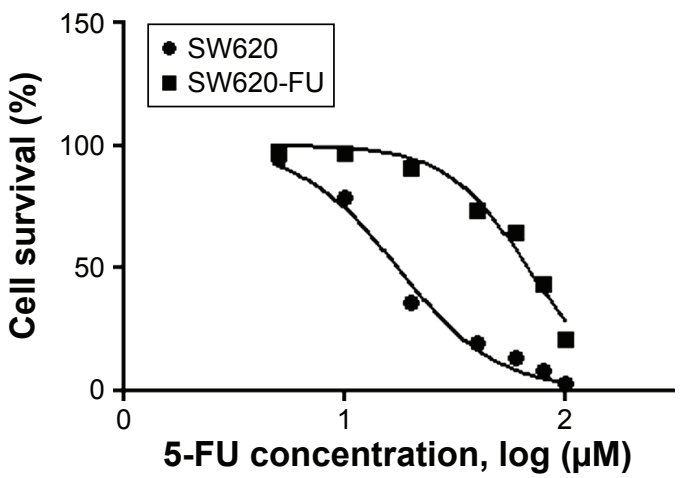

C

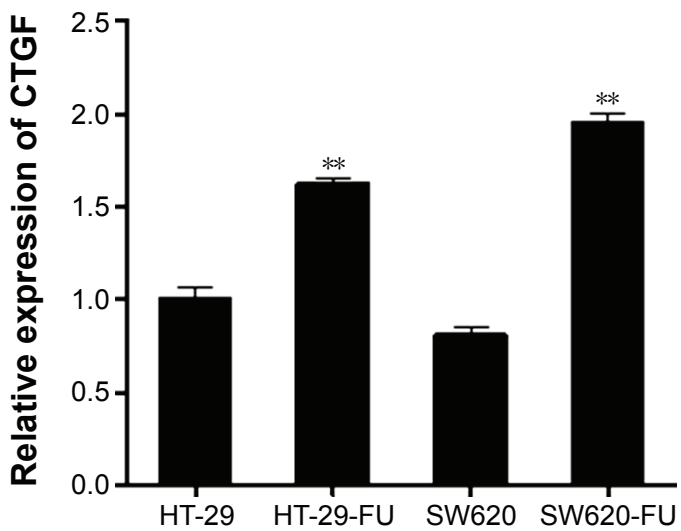

Figure I The expression of CTGF in human CRC cells.

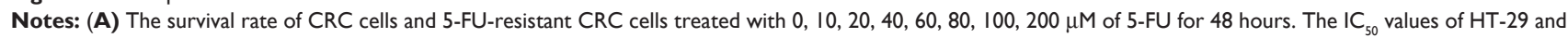
HT-29-FU were $14.2 \mathrm{I}$ and $66.7 \mathrm{I} \mu \mathrm{M}$ and the $\mathrm{IC}_{50}$ values of SW620 and SW620-FU were 17.46 and $67.86 \mu \mathrm{M}$, respectively. The protein and mRNA expression of CTGF in CRC cells and 5-FU-resistant CRC cells were examined by Western blotting (B) and qPCR (C). Statistical analyses results are expressed as mean \pm SEM. $* * P<0.0$ I as compared with control group.

Abbreviations: CTGF, connective tissue growth factor; CRC, colorectal cancer; qPCR, quantitative polymerase chain reaction; SEM, standard error of the mean; 5-FU, 5-fluorouracil.

These results revealed that upregulation of CTGF promoted resistance to 5-FU in human CRC 5-FU resistance cells, overexpression of CTGF protected 5-FU-induced cell death (Figure 2F and G). Therefore, CTGF expression is associated with a chemoresistant phenotype of CRC cells. Next, to examine why inhibition of CTGF reduced resistance to 5-FU, the effects of CTGF on cell cycle were examined. We found that downregulation of CTGF in CRC 5-FU resistance cells blocked the cell cycle at the G1 phase (Figure 3A-D). These data suggest that CTGF plays an important role in increasing the resistance of CRC cells to 5-FU.

\section{$\mathrm{Bcl}-\mathrm{xL}$ and survivin are involved in CTGF-mediated chemoresistance}

Mitochondrial pathway plays a central role in the regulation of 5-FU-mediated apoptotic cell death. ${ }^{11} \mathrm{Bcl}-\mathrm{xL}$ is an antiapoptotic member of the Bcl-2 family, which is located at the outer mitochondrial membrane and regulates outer mitochondrial membrane channel opening. To further investigate whether mitochondrial signaling is involved in CTGF-mediated resistance, Bcl-xL protein expression was examined. We found that overexpression of CTGF upregulates Bcl-xL expression (Figure 4A and B). Survivin is the tiniest member of inhibitors-of-apoptosis gene family, and the expression of survivin has been reported to be a marker of poor prognosis, which is selectively expressed in various kinds of benign and malignant tissues, but not in normal tissue. ${ }^{12,13}$ Overexpression of CTGF also upregulated survivin expression in human CRC (Figure 4A and B). According to these results, $\mathrm{Bcl}-\mathrm{xL}$ and survivin are important downstream factors in CTGF and enhance the sensitivity of CRC cells to 5 -FU. 
A

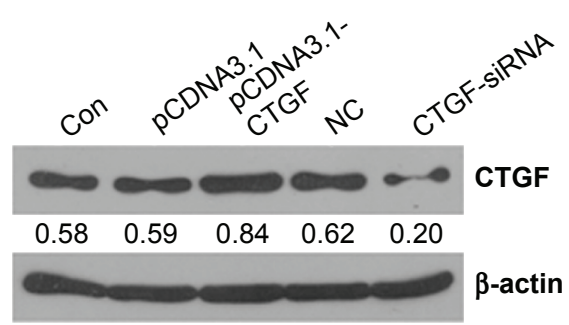

C

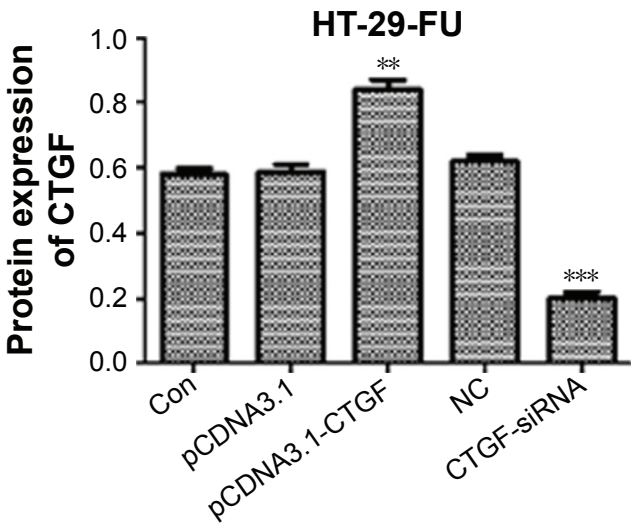

B

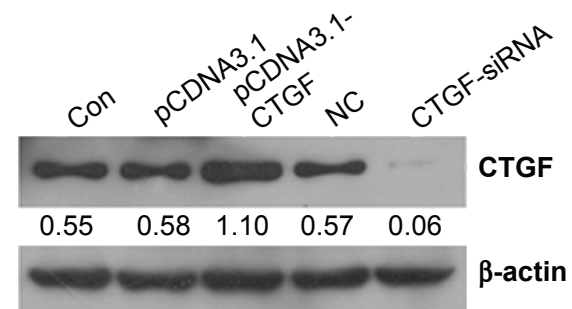

D

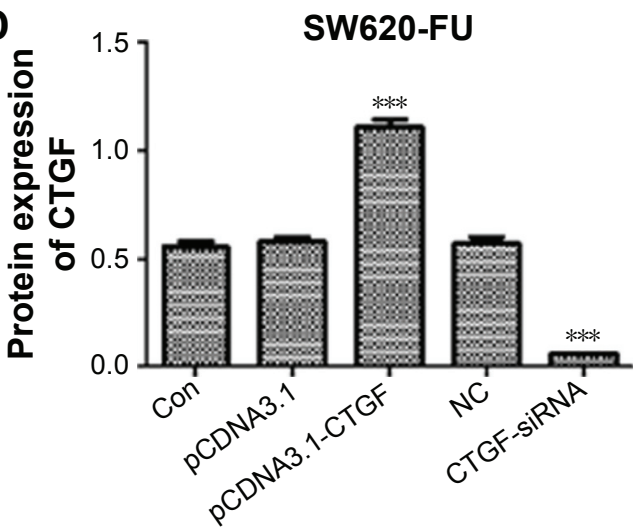

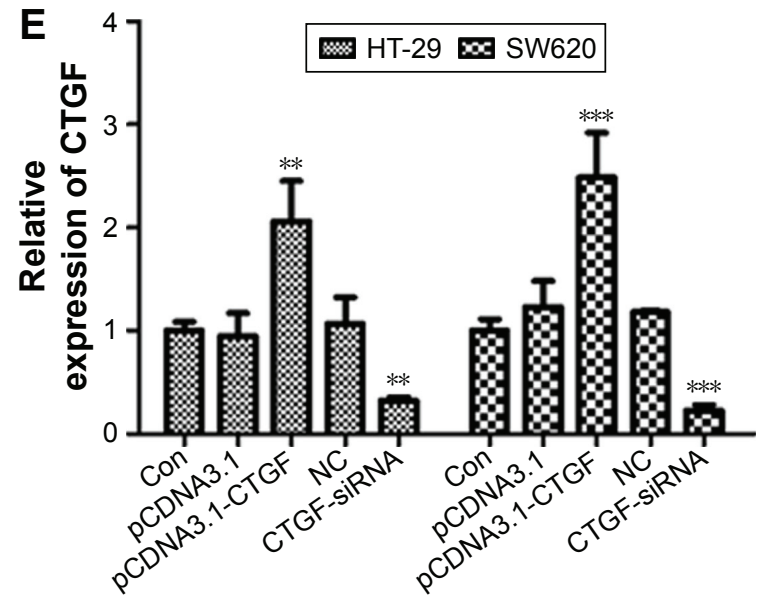
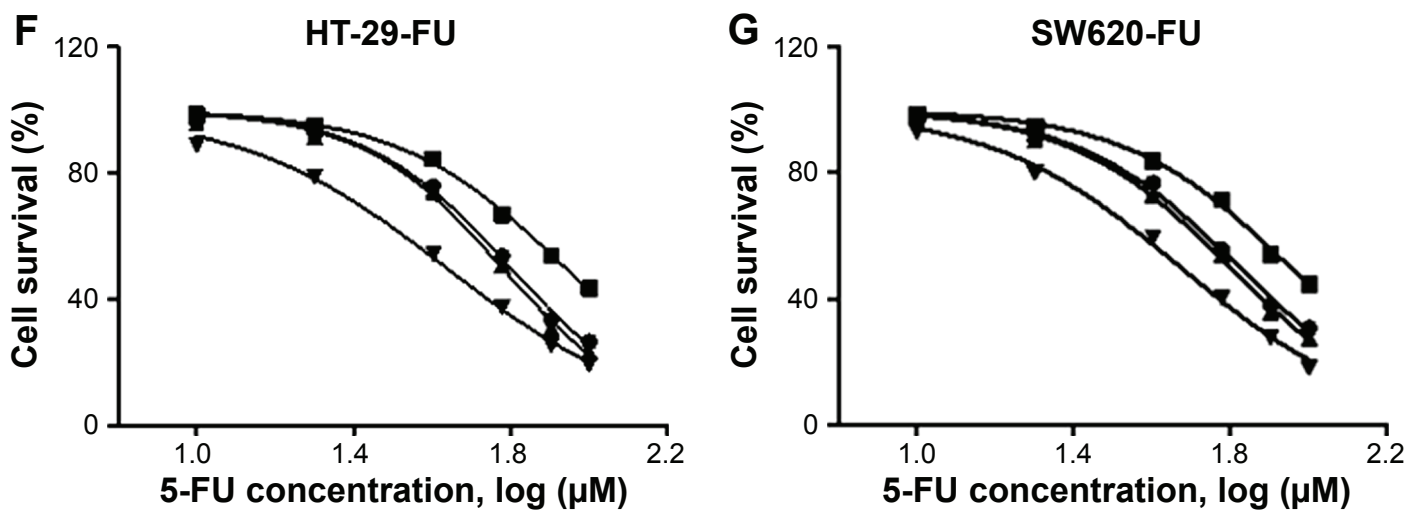

- pCDNA3.1 aCDNA3.1-CTGF $\Delta$ NC $\quad \boldsymbol{\nabla}$ CTGF-SiRNA

Figure 2 Construction and transfection of recombinant expression plasmids.

Notes: HT-29-FU and SW620-FU CRC cells were transfected with control, PCDNA3.I-CTGF vector, NC, CTGF-siRNA, and CTGF expression was examined by Western blotting (A-D) and qPCR (E). The cell viability was examined by MTT (F and $\mathbf{G})$. The IC $_{50}$ of pCDNA3.I, PCDNA3.I-CTGF, NC, CTGF-siRNA transfected group were 62.97, 86.77, 59.90, $43.27 \mu \mathrm{M}$ in HT-29-FU cells and 66.46, 89.50, 62.87, $47.05 \mu \mathrm{M}$ in HT-29-FU cells, respectively. Each experiment was repeated three times. Statistical analyses results are expressed as mean \pm SEM. $* * P<0.01$, $* * * P<0.00 \mathrm{I}$ compared with the control group.

Abbreviations: Con, control; CTGF, connective tissue growth factor; CRC, colorectal cancer; IC 5 , $50 \%$ inhibitive concentration; siRNA, small interfering RNA; SEM, standard error of the mean; qPCR, quantitative polymerase chain reaction; 5-FU, 5-fluorouracil; NC, negative control. 
CTGF activates MEK/ERK survival signaling pathways to subsequently protect 5-FU-induced cell apoptosis

Resistance to chemotherapy drugs not only can reduce the rate of cell apoptosis but also promote cell survival. ${ }^{14}$
FAK-dependent MEK/ERK activation is a common survival signaling pathway. ${ }^{15}$ We examined whether CTGF-mediated FAK, MEK, and ERK signal pathway or PI3K/AKT signal pathway activation promoted cell survival during 5-FU treatment. We found that the expression of $\mathrm{p}-\mathrm{MEK} / \mathrm{ERK}$
A

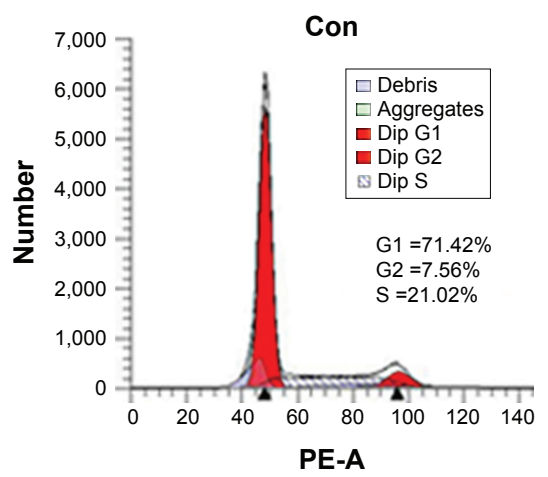

HT-29-FU

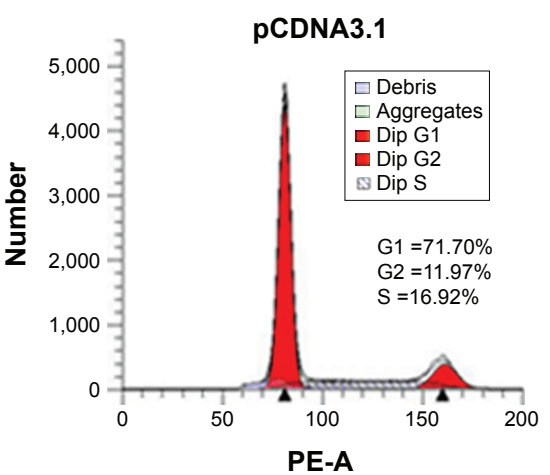

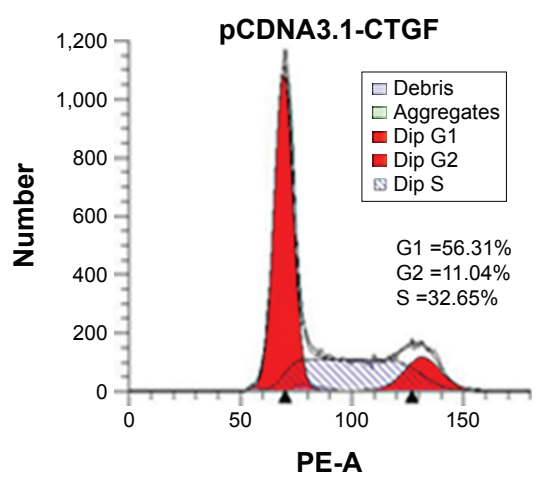

CTGF-siRNA
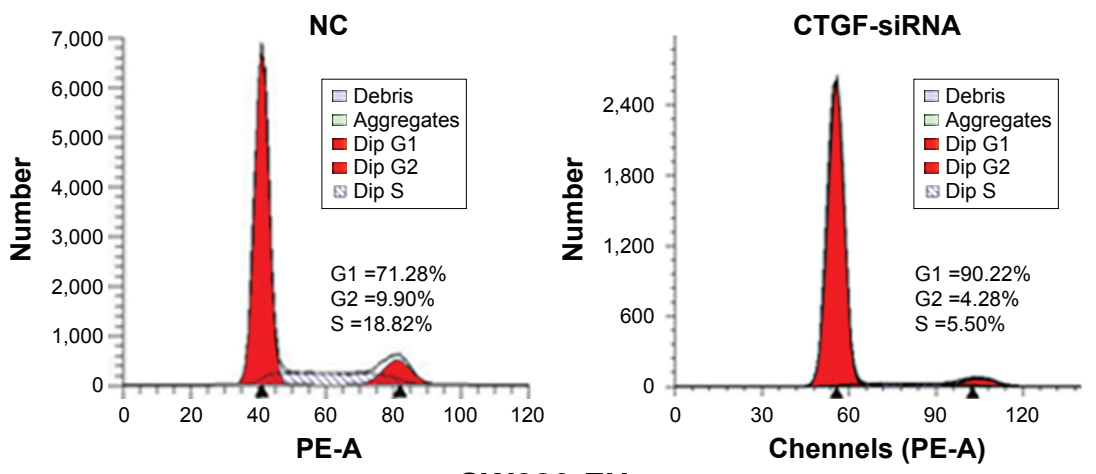

B

\section{SW620-FU}
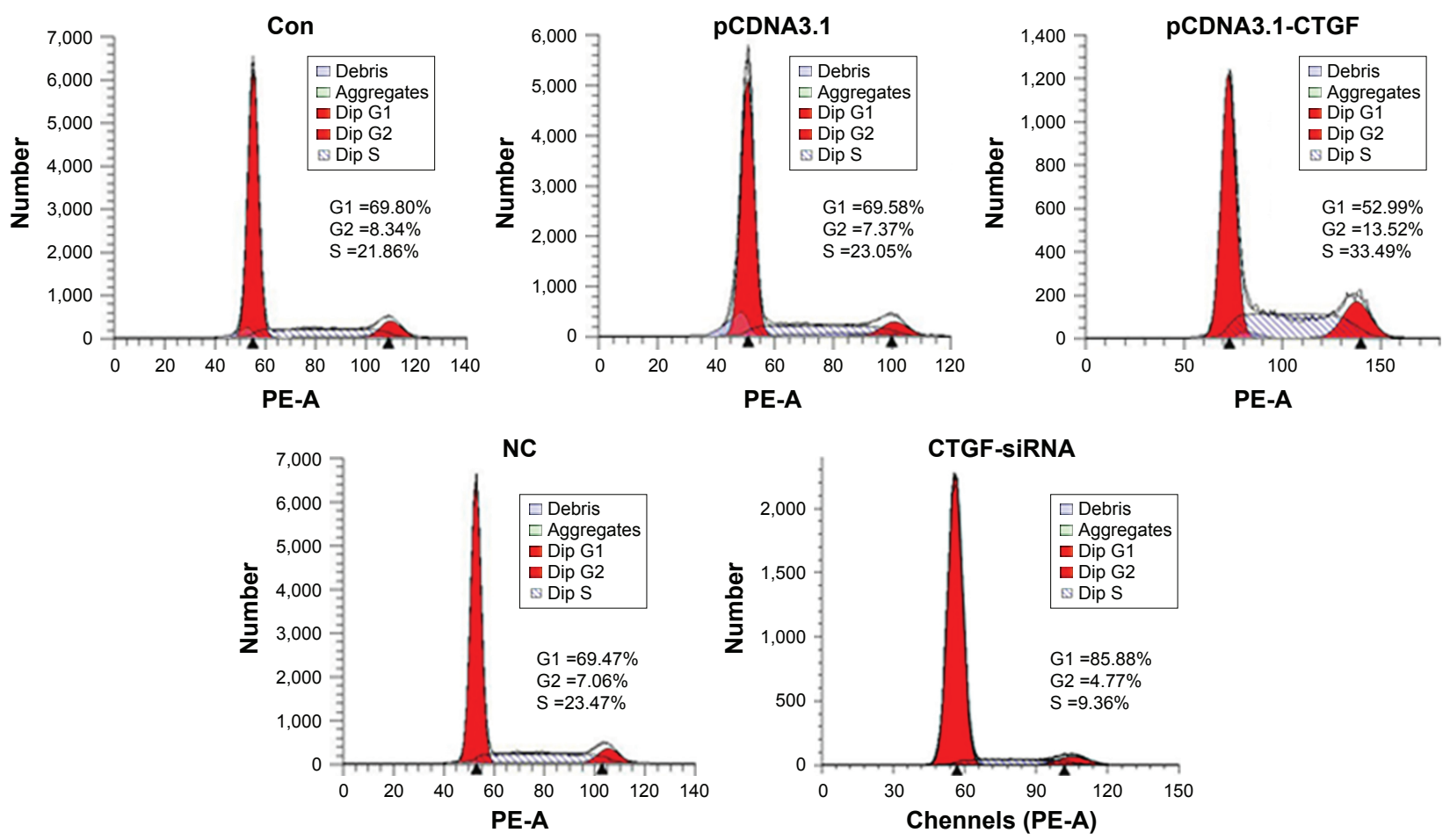

Figure 3 (Continued) 

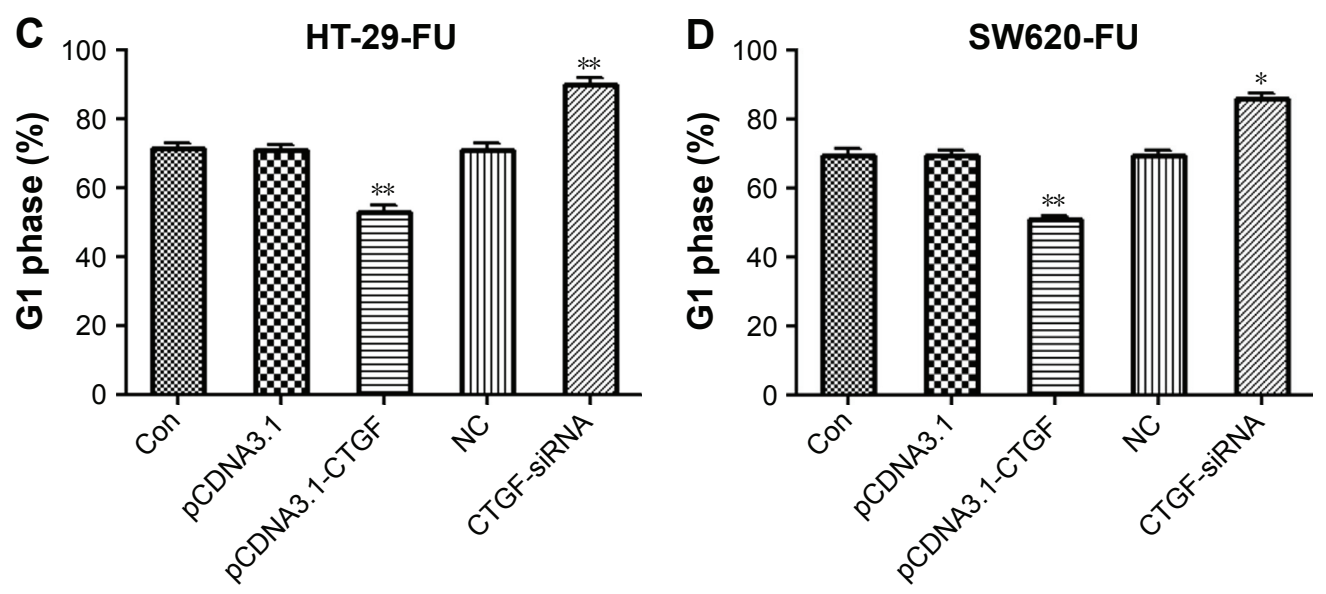

Figure 3 Downregulation of CTGF enhances cell cycle blocked in GI phase.

Notes: HT-29-FU and SW620-FU CRC cells were transfected with control, PCDNA3.I-CTGF, NC, CTGF-siRNA vectors. Cells were treated with 5-FU for 48 hours, and the effects of CTGF on cell cycle were examined (A-D). Each experiment was repeated three times. Statistical analyses results are expressed as mean \pm SEM. $* P<0.05$, $* * P<0.01$ as compared with control group.

Abbreviations: Con, control; CTGF, connective tissue growth factor; CRC, colorectal cancer; siRNA, small interfering RNA; SEM, standard error of the mean; 5-FU, 5 -fluorouracil; NC, negative control.

A

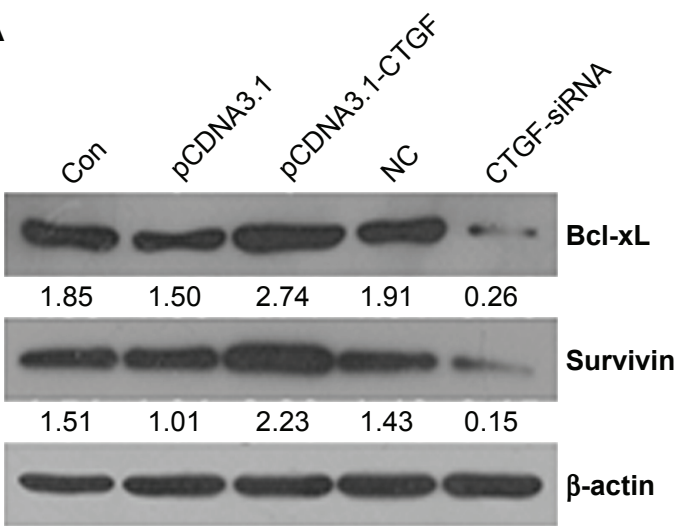

HT-29-FU

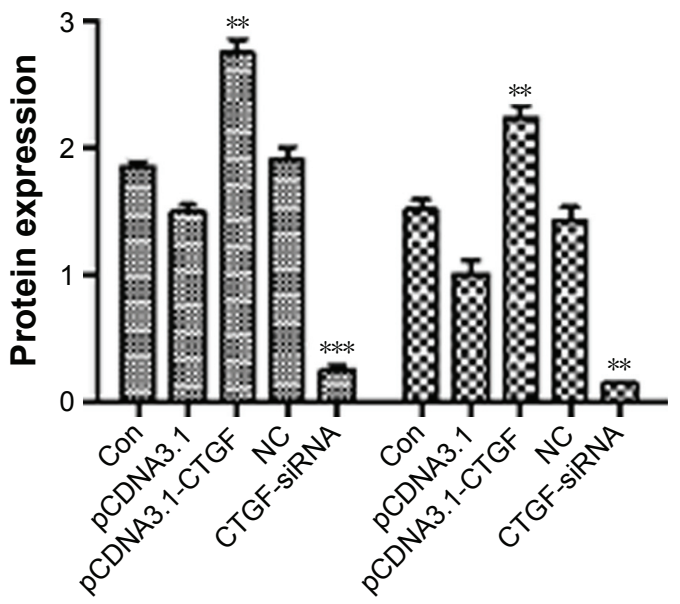

B
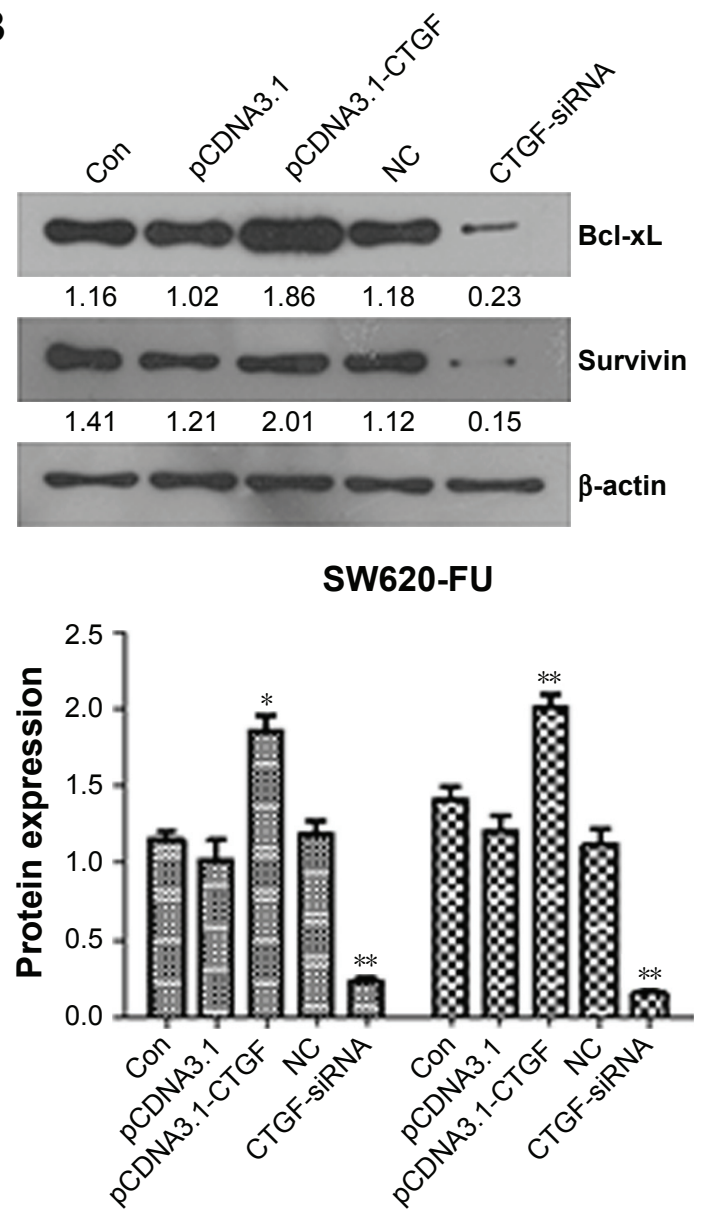

Bcl-xL Survivin

Figure $4 \mathrm{Bcl}-\mathrm{xL}$ and survivin are involved in CTGF-mediated chemoresistance.

Notes: HT-29-FU and SW620-FU CRC cells were transfected with control, pCDNA3.I-CTGF, NC, CTGF-siRNA vectors. (A) Protein expressions of HT-29-FU were examined by Western blotting. (B) Protein expressions of SW620-FU were examined by Western blotting. Each experiment was repeated three times. Statistical analyses results are expressed as mean $\pm S E M$. $* P<0.05$, $* * P<0.01$, $* * * P<0.001$ as compared with control group.

Abbreviations: $\mathrm{Bcl}-\mathrm{xL}$, B-cell lymphoma-extra large; Con, control; CTGF, connective tissue growth factor; CRC, colorectal cancer; SEM, standard error of the mean; 5-FU, 5-fluorouracil; NC, negative control. 
and FOXM1 significantly increased (Figure 5A, C-G), and $\mathrm{p}-\mathrm{PI} 3 \mathrm{~K}$ and $\mathrm{p}-\mathrm{AKT}$ protein had no significant change in chemoresistant cell lines and parental cells detected by Western blot. These findings revealed that overexpression of CTGF did not activate other survival signaling molecules, such as PI3K/Akt and RhoA (Figure 5B, G-I).
We pretreated with FAK/MEK/ERK inhibitor (PD98059) and PI3K/AKT inhibitor (LY294002) in 5-Fu resistance cells, and transfected the plasmid of pCDNA3.1-CTGF to above cells. The CTGF-mediated chemoresistance of CRC cells were evaluated by Western blot and MTT assay. Western blot results showed PD98059 inhibitor dramatically

A

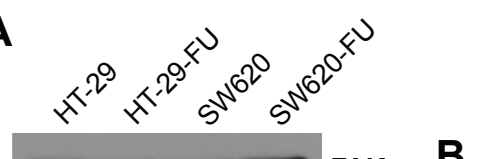

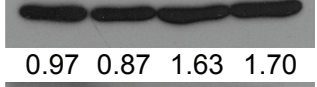

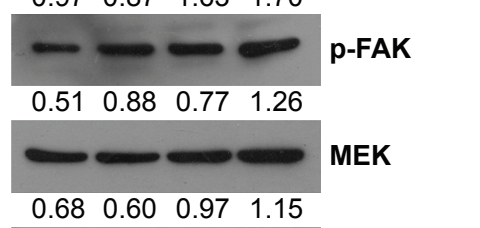

$\begin{array}{lllll}0.68 & 0.60 & 0.97 & 1.15 \\ - & 0 . M E K\end{array}$

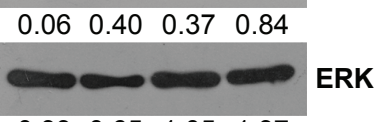

$0.93 \quad 0.65 \quad 1.05 \quad 1.37$
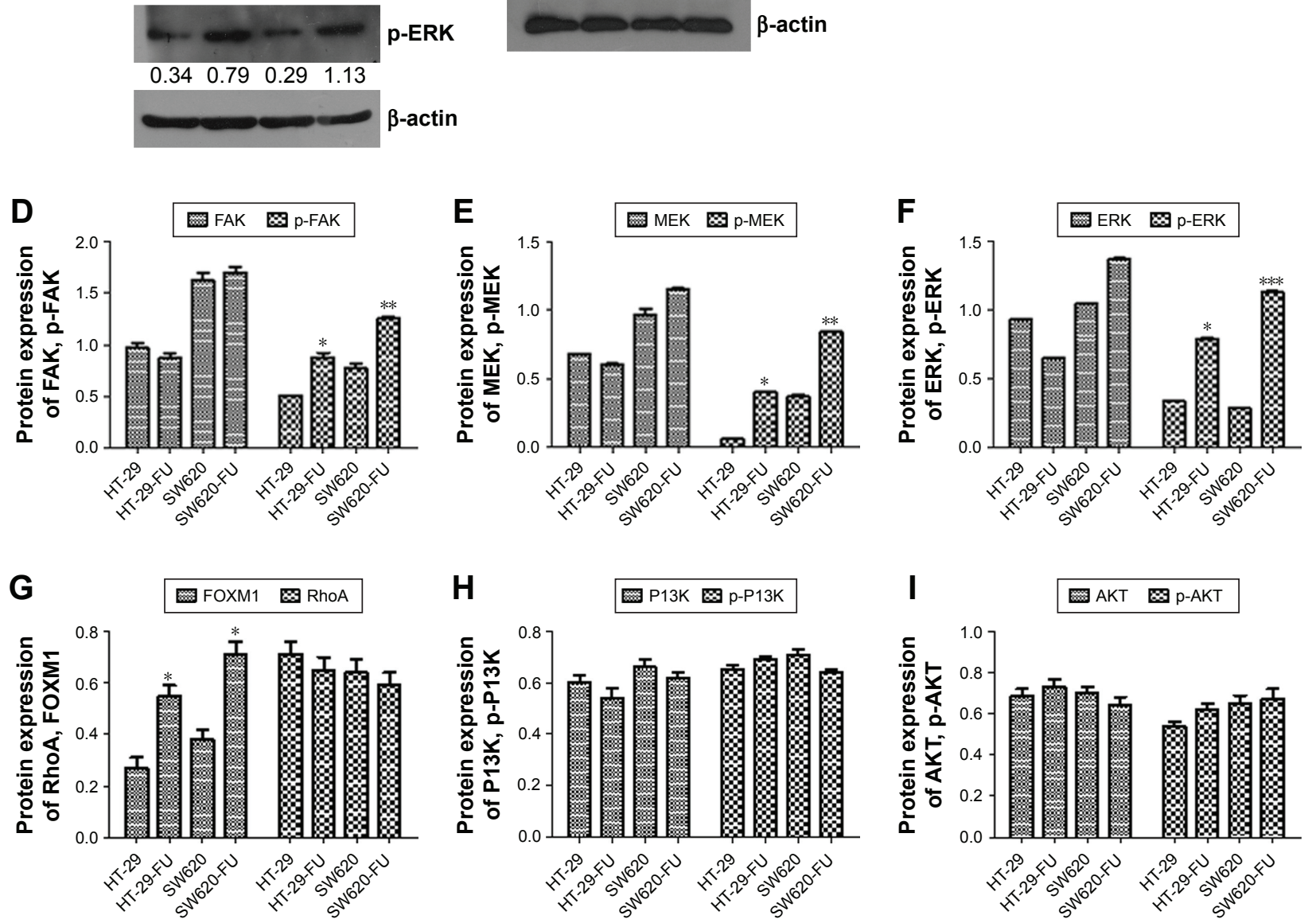

Figure 5 CTGF activates FAK/MEK/ERK survival signaling pathways during chemoresistance.

Notes: The protein of FAK/MEK/ERK (A and D-F), PI3K/AKT (B, H and I), FOXMI (C and $\mathbf{G})$ signal pathway were examined by Western bloting in parent and resistance cells of HT-29 and SW620. Each experiment was repeated three times. Statistical analyses results are expressed as mean \pm SEM. $* P<0.05$ as compared with control group. $* * P<0.01$. *** $P<0.001$.

Abbreviations: CTGF, connective tissue growth factor; SEM, standard error of the mean. 
decreased the apoptosis related protein expression of Bcl-xL and survivin (Figure 6A-C), and there was no significant changes in LY294002 treated groups. The MTT assay showed the $\mathrm{IC}_{50}$ of $\mathrm{FAK} / \mathrm{MEK} / \mathrm{ERK}$ inhibitor treated groups (HT-29-FU: $44.46 \mu \mathrm{M}$ and SW620-FU: $53.68 \mu \mathrm{M}$ ) was lower than control groups (HT-29-FU: $85.52 \mu \mathrm{M}$ and SW620-FU: $89.10 \mu \mathrm{M}$ ) in HT-29-FU and SW620-FU cells with CTGF overexpression. The LY294002 inhibitor
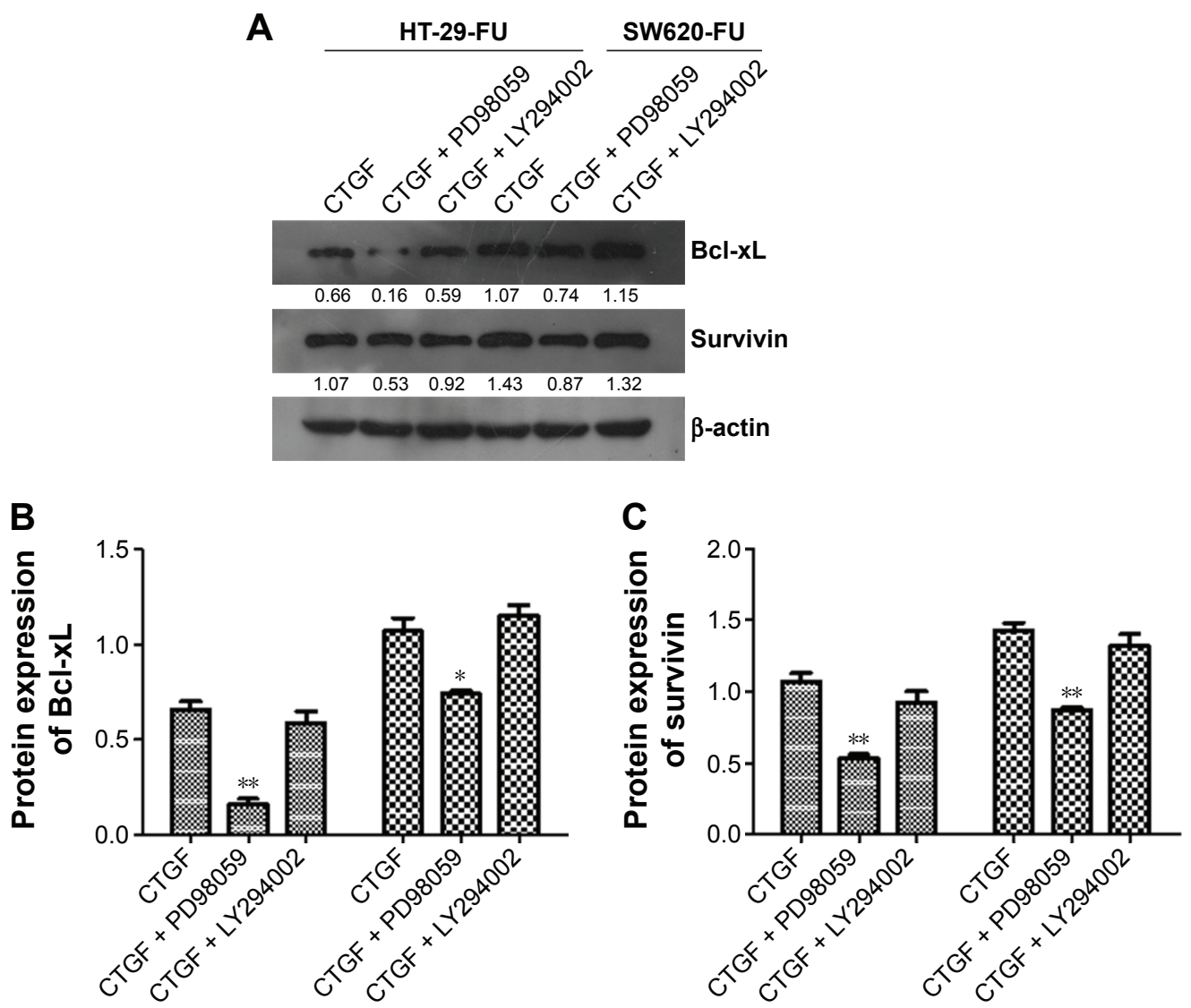

$\otimes \mathrm{HT}-29-\mathrm{FU} \quad \infty \mathrm{SW} 620-\mathrm{FU}$

D

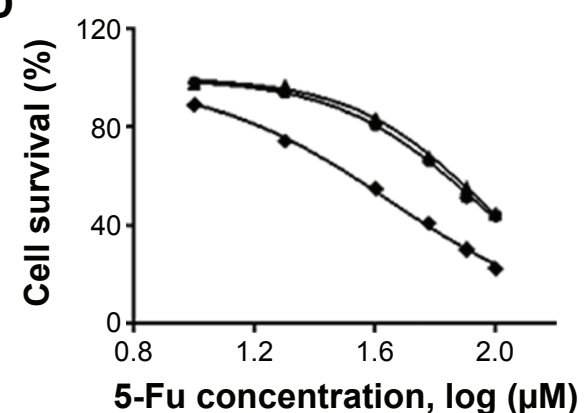

E

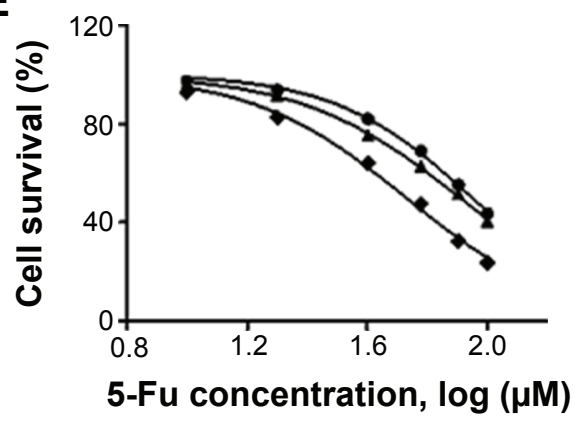

- pcDNA3.1-CTGF ^ pcDNA3.1-CTGF + LY294002 • pcDNA3.1-CTGF + PD98059

Figure 6 FAK/MEK/ERK inhibitor promoted 5-FU-induced cell death.

Notes: HT-29-FU and SW620-FU CRC cells were transfected with control or PCDNA3.I-CTGF vector. (A-C) Bcl-xL and survivin protein expressions were examined after MEK/ERK (PD98059) or PI3K/Akt inhibitor (LY294002) treated. (D and E) Cells were pretreated with PD98059 (I0 mM) or LY294002 (I0 mM), followed by stimulation with 5-FU for 48 hours. Cell survival ability and apoptosis were examined by MTT assay. The IC ${ }_{50}$ of PD98059 treated groups was $44.46 \mu M$ (HT-29-FU) and 53.68 $\mu$ M (SW620-FU). The IC I0 $_{50}$ of control groups was 85.52 and $89.10 \mu \mathrm{M}$ in HT-29-FU and SW620-FU cells, respectively, with CTGF overexpression. The IC 50 of LY294002 inhibitor treatment groups was $89.05 \mu \mathrm{M}$ (HT-29-FU) and $81.10 \mu \mathrm{M}$ (SW620-FU). Each experiment was repeated three times. Statistical analyses results were expressed as mean \pm SEM. $* P<0.05$ as compared with control group. ${ }^{*} * P<0.01$.

Abbreviations: Bcl-xL, B-cell lymphoma-extra large; CTGF, connective tissue growth factor; CRC, colorectal cancer; SEM, standard error of the mean; 5-FU, 5-fluorouracil. 
treatment groups (HT-29-FU: $89.05 \mu \mathrm{M}$ and SW620-FU: $81.10 \mu \mathrm{M})$ showed no significant difference compared with control groups. These results indicated that FAK/MEK/ERK inhibitor promoted 5-FU-induced cell death, and the PI3K/ AKT inhibitor had no effect (Figure 6D and E). Therefore, our results suggest that FOXM1 contributes to 5-FU resistance downstream of MEK/ERK signaling, subsequently conveying resistance to cell apoptosis.

\section{Discussion}

CRC remains one of the leading causes of morbidity and mortality worldwide with high disease incidence ${ }^{16}$ Chemotherapy is one of the main methods of treating CRC and occupies an important position. However, CRC cells are often less sensitive or gradually become resistant to the drugs. It is therefore important to research the function of drug resistance to chemotherapeutic agents. Previous studies have reported CTGF overexpression in CRC cells compared to adjacent tissues, and high CTGF expression inhibits metastasis and acts as an independent prognostic marker in CRC. ${ }^{8,17,18}$ Moreover, our analysis suggests that overexpressed CTGF in CRC cells increased the tolerance to 5-FU by 5 -FU-mediated apoptosis and facilitating carcinoma cell survival. The overexpression of CTGF specifically upregulated Bcl-xL and survivin expression. CTGF also promoted FAK, MEK, and ERK survival signal pathways to improve cell survival rate during 5-FU therapy. Taken together, we are of the opinion that CTGF plays an indispensable role in CRC development by supporting cancer cell survival and drug tolerance. To make sense of the relationship between CTGF and chemoresistance of CRC cells, the chemoresistant phenotype of CRC cells were constructed. Overexpression of CTGF defended 5-FU-mediated cell death in these CRC cells. Therefore, over expression of CTGF promoted the chemoresistance of CRC cells.

CTGF can regulate the activity of diverse growth factors or integrins. ${ }^{19,20}$ CTGF was found to activate the cellular proliferation, migration, and fibrogenesis. ${ }^{21}$ CTGF has also been proven to participate in certain types of cancers of drug-resistance. For instance, in human osteosarcoma, overexpression of CTGF significantly increased the resistance to cisplain- and paclitaxel-mediated cell apoptosis. ${ }^{22,23} \mathrm{In}$ glioblastoma multiforme, overexpression of CTGF caused the U343 glioblastoma multiforme cells to survive for longer than 40 days in serum-free medium and resist antitumor drugs, including tumor necrosis factor, tumor necrosis factor-related apoptosis-inducing ligand, VELCADE (bortezomib, proteasome inhibitor), and temozolomide. ${ }^{4} \mathrm{CTGF}$ also suppresses cell growth and resistance to apoptosis on exposure to doxorubicin and paclitaxel in breast cancer. ${ }^{6}$ However, the function of CTGF is undiscovered in human CRC drug-resistance. In this study, using Western blot, quantitative PCR, and cell cycle analysis, we found that 5-FU enhanced CRC cell death through an apoptotic mechanism. We also found that overexpression of CTGF enhanced the tolerance to 5-FU-induced cell apoptosis. Our study has provided the important evidence that CTGF provides intensive chemoresistance to 5-FU in CRC. As a consequence, CTGF may be a novel chemical therapy target in CRC.

Survivin has been found overexpressed in bladder, lung, liver carcinoma, and metrocarcinoma cancers, ${ }^{24}$ and inhibited tumor formation and angiogenesis in some cancers. ${ }^{25}$ So, survivin plays an important role in inhibiting the apoptosis pathway and sensitizing cancer cells to chemotherapeutic agents. ${ }^{25,26} \mathrm{Bcl}-\mathrm{xL}$ is a member of $\mathrm{Bcl}-2$ protein family and decrease in $\mathrm{Bcl}-\mathrm{xL}$ expression is necessary for treating apoptosis and increasing chemosensitivity in tumor cells. ${ }^{27}$ In our research, we found that CTGF enhanced the expression of Bcl-xL and survivin in CRC drug-resistance cells. Furthermore, PD98059 inhibitor treated in CTGF overexpressing CRC drug-resistance cells inhibited the protein expression of $\mathrm{Bcl}-\mathrm{xL}$ and survivin. These results show that CTGF promoted resistances to 5 -Fu-mediating cell apoptosis by activating the defects in apoptotic pathway to produce resistance to apoptosis, and $\mathrm{Bcl}-\mathrm{xL}$ and survivin are critical regulating factors during this process.

FAK is a possible signal molecule that is involved in activating the integrin-mediated signal. ERK and MEK proteins are often upregulated in answer to DNA-damaging chemotherapeutants, such as 5 -FU..$^{28}$ Our study found that CTGF enhanced the activity of FAK, MEK, and ERK proteins. However, CTGF did not influence phosphorylation of PI3K and the Akt signaling cascade. Moreover, FAK and MEK inhibitors reversed CTGF-mediated resistance to 5-FU. It is our opinion that CTGF activated FAK, MEK, and ERK survival signal pathways and subsequently inhibited 5-FUmediated cell apoptosis in CRC.

In conclusion, we demonstrated that overexpressing CTGF significantly promoted chemoresistance in CRC cells, which decreased apoptosis and enhanced tolerance to 5-FU therapy, and downregulation of CTGF expression increased chemosensitivity of CRC cells. Thus, we conclude these results support an investigation of CTGF as a strategic target for CRC treatment, CTGF increases tolerance to 5-FU-induced cell apoptosis through FAK/MEK/ERK signal pathway in CRC. 


\section{Acknowledgment}

This work was supported by Science and Technology Project of Hunan Province (No 2015SK20206).

\section{Disclosure}

The authors report no conflicts of interest in this work.

\section{References}

1. Xie X, Liu H, Wang Y, et al. Nicotinamide N-methyltransferase enhances resistance to 5 -fluorouracil in colorectal cancer cells through inhibition of the ASK1-p38 MAPK pathway. Oncotarget. Epub 2016 Jun 13.

2. Mhaidat NM, Bouklihacene M, Thorne RF. 5-Fluorouracil-induced apoptosis in colorectal cancer cells is caspase-9-dependent and mediated by activation of protein kinase C-delta. Oncol Lett. 2014;8(2): 699-704.

3. Liu H, Yin Y, Hu Y, et al. miR-139-5p sensitizes colorectal cancer cells to 5-fluorouracil by targeting NOTCH-1. Pathol Res Pract. 2016;212(7): 643-649.

4. Yin D, Chen W, O'Kelly J, et al. Connective tissue growth factor associated with oncogenic activities and drug resistance in glioblastoma multiforme. Int J Cancer. 2010;127(10):2257-2267.

5. Lin BR, Chang CC, Chen RJ, et al. Connective tissue growth factor acts as a therapeutic agent and predictor for peritoneal carcinomatosis of colorectal cancer. Clin Cancer Res. 2011;17(10):3077-3088.

6. Wang MY, Chen PS, Prakash E, et al. Connective tissue growth factor confers drug resistance in breast cancer through concomitant upregulation of Bcl-xL and cIAP1. Cancer Res. 2009;69(8):3482-3491.

7. Cui L, Zhang Q, Mao Z, et al. CTGF is overexpressed in papillary thyroid carcinoma and promotes the growth of papillary thyroid cancer cells. Tumour Biol. 2011;32(4):721-728.

8. Ladwa R, Pringle H, Kumar R, West K. Expression of CTGF and Cyr61 in colorectal cancer. J Clin Pathol. 2011;64(1):58-64.

9. Gayle SS, Castellino RC, Buss MC, Nahta R. MEK inhibition increases lapatinib sensitivity via modulation of FOXM1. Curr Med Chem. 2013; 20(19):2486-2499.

10. Minn AJ, Kang Y, Serganova I, et al. Distinct organ-specific metastatic potential of individual breast cancer cells and primary tumors. $J$ Clin Invest. 2005;115(1):44-55.

11. Cepeda V, Fuertes MA, Castilla J, Alonso C, Quevedo C, Pérez JM. Biochemical mechanisms of cisplatin cytotoxicity. Anticancer Agents Med Chem. 2007;7(1):3-18.

12. Wang W, Luo H, Wang A. Expression of survivin and correlation with PCNA in osteosarcoma. J Surg Oncol. 2006;93(7):578-584.

13. Liang X, Da M, Zhuang Z, et al. Effects of Survivin on cell proliferation and apoptosis in MG-63 cells in vitro. Cell Biol Int. 2009;33(1): $119-124$
14. Gimenez-Bonafe P, Tortosa A, Perez-Tomas R. Overcoming drug resistance by enhancing apoptosis of tumor cells. Curr Cancer Drug Targets. 2009;9(3):320-340.

15. Kaufmann SH, Desnoyers S, Ottaviano Y, Davidson NE, Poirier GG Specific proteolytic cleavage of poly(ADP-ribose) polymerase: an early marker of chemotherapy-induced apoptosis. Cancer Res. 1993;53(17): 3976-3985.

16. Hammond WA, Swaika A, Mody K. Pharmacologic resistance in colorectal cancer: a review. Ther Adv Med Oncol. 2016;8(1):57-84.

17. Lin BR, Chang CC, Che TF, et al. Connective tissue growth factor inhibits metastasis and acts as an independent prognostic marker in colorectal cancer. Gastroenterology. 2005;128(1):9-23.

18. Guo Y, Li X, Lin C, et al. MicroRNA133b inhibits connective tissue growth factor in colorectal cancer and correlates with the clinical stage of the disease. Mol Med Rep. 2015;11(4):2805-2812.

19. Shakunaga T, Ozaki T, Ohara N, et al. Expression of connective tissue growth factor in cartilaginous tumors. Cancer. 2000;89(7): $1466-1473$.

20. Sodek KL, Ringuette MJ, Brown TJ. Compact spheroid formation by ovarian cancer cells is associated with contractile behavior and an invasive phenotype. Int J Cancer. 2009;124(9):2060-2070.

21. Hamada S, Masamune A, Shimosegawa T. Novel therapeutic strategies targeting tumor-stromal interactions in pancreatic cancer. Front Physiol. 2013;4:331.

22. Tsai HC, Huang CY, Su HL, Tang CH. CCN2 enhances resistance to cisplatin-mediating cell apoptosis in human osteosarcoma. PloS One. 2014;9(3):e90159

23. Tsai HC, Huang CY, Su HL, Tang CH. CTGF increases drug resistance to paclitaxel by upregulating survivin expression in human osteosarcoma cells. Biochim Biophys Acta. 2014;1843(5):846-854.

24. Karczmarek-Borowska B, Filip A, Wojcierowski J, Smoleń A, Pilecka I, Jabłonka A. Survivin antiapoptotic gene expression as a prognostic factor in non-small cell lung cancer: in situ hybridization study. Folia Histochem Cytobiol. 2005;43(4):237-242.

25. Garg H, Suri P, Gupta JC, Talwar GP, Dubey S. Survivin: a unique target for tumor therapy. Cancer Cell Int. 2016;16:49.

26. Ambrosini G, Adida C, Altieri DC. A novel anti-apoptosis gene, survivin, expressed in cancer and lymphoma. Nature Med. 1997;3(8):917-921.

27. Tsai HC, Su HL, Huang CY, Fong YC, Hsu CJ, Tang CH. CTGF increases matrix metalloproteinases expression and subsequently promotes tumor metastasis in human osteosarcoma through downregulating miR-519d. Oncotarget. 2014;5(11):3800-3812.

28. Spina A, Sorvillo L, Di Maiolo F. Inorganic phosphate enhances sensitivity of human osteosarcoma U2OS cells to doxorubicin via a p53-dependent pathway. J Cell Physiol. 2013;228(1):198-206.
OncoTargets and Therapy

\section{Publish your work in this journal}

OncoTargets and Therapy is an international, peer-reviewed, open access journal focusing on the pathological basis of all cancers, potential targets for therapy and treatment protocols employed to improve the management of cancer patients. The journal also focuses on the impact of management programs and new therapeutic agents and protocols on

\section{Dovepress}

patient perspectives such as quality of life, adherence and satisfaction. The manuscript management system is completely online and includes a very quick and fair peer-review system, which is all easy to use. Visit http://www.dovepress.com/testimonials.php to read real quotes from published authors. 